\title{
"Dios nos mejore las horas si nos conviene": La salud y la enfermedad de una familia montañesa del siglo XIX a través del estudio de las escrituras del yo ${ }^{1}$
}

\author{
"God Improves our Hours if he Agrees": Health \\ and Disease of a Cantabrian Family in the Nineteenth \\ Century through the Study of the Egodocuments
}

Carmen Rubalcaba Pérez
Red de Archivos e Investigadores de la Escritura Popular

(RedAIEP)

RESUMEN

A partir del nacimiento, en los años setenta del siglo xx, de la denominada antropología de la salud y la enfermedad, se inició el reconocimiento de ambos estados como fenómenos culturales y los antropólogos comenzaron a sumergirse en la comprensión de la manera en que se elaboran los conceptos de sano y enfermo. La historia social de la cultura escrita, a través del estudio de los testimonios escritos del pasado, se constituye en un recurso insustituible para analizar las dimensiones sociales y culturales del proceso de enfermar en su dimensión histórica. A través del análisis de la documentación autógrafa de carácter privado - correspondencia y anotaciones personales en libros de cuentas- de un pequeño propietario rural acomodado, Pedro Jado Agüero (18151891), se muestra cómo la enfermedad no consiste sólo en una disfunción biológica, sino también en una matriz de significados culturalmente establecidos que hay que intentar desentrañar. Descubrir el sentido simbólico de la enfermedad a través del análisis del conjunto de experiencias, de palabras y de sentimientos utilizados o descritos por los integrantes de una cultura significa acercarse en mayor medida al conocimiento de su estructura de significados.

Palabras clave: Salud, Discurso, Narración, Religiosidad popular, Memoria, Cartas.

${ }^{1}$ La autora desea expresar su profundo agradecimiento a los dos evaluadores que revisaron la versión anterior de este trabajo. Su lectura fue atenta y cuidadosa y su crítica ayudó a mejorarlo. Evidentemente, cualquier error o incorrección que persista en el texto es responsabilidad exclusiva de la autora. 


\section{SUMMARY}

From birth in the seventies of the twentieth century of the so-called Anthropology of Health and Disease, the recognition of health and disease as cultural phenomena was introduced and anthropologists began to deepen in understanding how to develop the concepts of healthy and sick. The social history of literacy through the study of written records of the past, constitutes an irreplaceable resource for analyzing the social and cultural dimensions of disease process in its historical dimension. Through the analysis of private handwritten documents - personal correspondence and records in accounting books - of a small wealthy landowner, Pedro Jado Agüero (1815-1891), we know how the disease is not just a biological dysfunction, but also an array of culturally established meanings that we must try to unravel. Discovering the symbolism of the disease through the analysis of all the experiences, words and feelings used or described by members of a culture means to move further to the knowledge of the structure of meanings of the same culture.

Key words: Health, Discourse, Narration, Popular Religion, Memory, Letters.

\section{INTRODUCCIÓN}

Desde que en los años treinta, Henry Sigerist comenzase su proyecto de renovación de la historia médica entonces vigente hasta la actualidad, la historiografía médica ha experimentado grandes cambios (Arrizabalaga 1992: 149; López Piñero y Terrada Ferrandis 2000: 61). Sigerist fue uno de los primeros historiadores de la medicina que, siguiendo el camino de la medicina social abierto por Virchow, mostró las relaciones entre la práctica médica y las condiciones sociales.

Sus planteamientos fueron desarrollados y profundizados por la siguiente generación de historiadores de la medicina entre los que destaca Erwin $\mathrm{H}$. Ackerknecht que, además de ser discípulo de Sigerist, se había formado como antropólogo en París junto a Marcel Mauss (López Piñero y Terrada Ferrandis 2000: 62-63). Como consecuencia de lo anterior, se puede afirmar que en los años setenta del siglo xx en la historiografía de la enfermedad y, en general, de la medicina se produjo la ansiada renovación propuesta por Sigerist décadas atrás (Arrizabalaga 1992: 149), se inició el reconocimiento de la salud y de la enfermedad como fenómenos culturales y los antropólogos comenzaron a adentrarse en la comprensión de la manera en que se elaboran los conceptos de salud y enfermedad, de sano y enfermo ${ }^{2}$.

${ }^{2}$ En España, por ejemplo, Luis García Ballester comenzó en los años setenta del siglo pasado a abordar en sus trabajos un tema que entonces comenzaba a tratar la antropología médica: la consideración de la medicina como una forma cultural (Comelles 2002: 489). 
A través del proceso de socialización, el individuo adquiere unos criterios morales que le hacen ser aceptado dentro de su grupo social, entre esos valores se encuentran las diferentes formas de representación de la enfermedad $^{3}$, cuya imagen construye cada grupo humano según un modelo explicativo que se adapta a su estructura social y su cosmovisión, a su Weltanschauung particular.

La finalidad de este trabajo, sin embargo, no reside en demorarse en la evolución de los conceptos de salud y enfermedad como construcciones sociales, campo sobre el que ya existe un amplio corpus bibliográfico ${ }^{4}$, sino apuntar cómo la historia social de la cultura escrita, a través del estudio de los testimonios escritos del pasado, se constituye en un recurso insustituible para analizar las dimensiones sociales y culturales del proceso de enfermar en su devenir histórico, mostrar la cultura médica de una familia concreta en el pasado y conocer las categorías usadas por los actores históricos a través de sus propias palabras.

\section{LA CORRESPONDENCIA COMO FUENTE PARA EL ANÁLISIS HISTÓRICO}

Las correspondencias ordinarias —de la gente común- han sido consideradas a menudo banales y repetitivas. Sin embargo, permiten acceder a aspectos de la vida humana que desde el punto de vista histórico han resultado siempre prácticamente inalcanzables — la vida emocional, personal, íntima de las gentes que han construido, inconscientemente, la historia- y su estudio proporciona indicios de las relaciones sociales, del pensamiento y de la ideología del momento en que fueron producidas. Desde una perspectiva antropológica, la correspondencia familiar puede ser entendida como el producto de una práctica ritualizada en la cual los individuos, enfrentados a un conjunto de referencias y de modelos, deben clasificar la realidad y reevaluar sus relaciones con los otros (Dauphin, Lebrun-Pézerat y Poublan 1995: 99).

\footnotetext{
${ }^{3}$ Sobre el concepto de socialización ha escrito Arthur Kleinman: "The individual absorbs (internalizes) social reality —as a system of symbolic meanings and norms governing his behaviour, his perception of the world, his communication with others, and his understanding of both the external, intrapsychic space- during the process of socialization (or enculturation). Socialization takes place in the family, but also in other social groupings via education, occupation, rituals, play, and the general process of internalizing norms from the world we live in". (Kleinman 1980: 35).

${ }^{4}$ Sin pretensión de exhaustividad, se pueden citar los siguientes autores: Sigerist 1943; Rosen 1943; Ackerknecht 1945, 1963, 1971 y 1982; Porter 1983 y 1985; Porter y Wear 1987; Duden 1991.
} 
Dentro de los distintos conjuntos epistolares, la correspondencia familiar posee una serie de características que la individualizan y la diferencian como, por ejemplo, la existencia de una red familiar, las formas de conservación, los recursos retóricos empleados y su finalidad que hace de ella un instrumento de unión y solidaridad (Dauphin; Lebrun-Pézerat; Poublan 1995: 192; Anderson 1988; Burguière y otros 1988). La carta, mejor que ninguna otra expresión, aúna el lazo social y la subjetividad (Chartier 1991: 9). La correspondencia significa, como cualquier otra forma de relación con los demás, la conquista de una posición desde la cual el individuo se relaciona con el grupo y la forja de una identidad personal y social a través de la utilización de las fórmulas de articulación socialmente elaboradas y codificadas.

CARACTERÍSTICAS DE LA DOCUMENTACIÓN PRIVADA DE LA FAMILIA JADO: PRODUCCIÓN Y CONSERVACIÓN

En pocas ocasiones puede el investigador hallar un conjunto coherente, continuo y rico de documentación autógrafa de carácter privado como el que aquí se presenta. Se trata de la correspondencia y los libros de cuentas con anotaciones personales y familiares de un pequeño propietario rural acomodado, Pedro Jado Agüero (1815-1891), que vivió en el pueblo montañés de Escalante y en la vecina localidad de Santoña en el siglo XIX 5 .

La correspondencia de la familia Jado a lo largo del siglo XIX y primeros años del xx pivota en torno a la figura de Pedro Jado, verdadero eje a cuyo alrededor se articulan todas las relaciones epistolares o, al menos, las que se han conservado ${ }^{6}$. Sus hijos se dirigen a él, incluyendo en las cartas saludos, mensajes y recados para su madre, Joaquina Ocejo, y resto de hermanos.

Pedro Jado Agüero nació en Escalante el 28 de abril de 1815. Fueron sus padres José Jado Valle (1798-1864) y Ma Dolores Agüero Valle (17931877). Tuvo otros cuatro hermanos: Casimiro, Josefa, Petra y Ventura. Con 17 años emigró a Cuba, en 1832, permaneciendo en La Habana hasta 1839. De regreso en Escalante, en el año 1840 contrajo matrimonio con Joaquina Ocejo Castanedo (1823-1902), unión de la que nacieron doce hijos, algunos - Gregorio, Bernardino, Nicomedes - fallecidos en edad muy temprana. De los que sobrevivieron y alcanzaron la edad adulta - Atanasia, Julia, Emilio,

\footnotetext{
${ }^{5}$ El libro de cuentas utilizado en esta ocasión es el mantenido por Pedro Jado Agüero entre el 19 de noviembre de 1844 y el cuatro de abril de 1857.

${ }^{6}$ La documentación de la familia Jado se conservó en un primer momento en el archivo familiar de San Román de Escalante y en la actualidad en el archivo privado del Palacio de los Condes de Isla-Fernández en Isla (Cantabria).
} 
Mariano, Ezequiel, Joaquina, Alberto, Darío y Ventura-, hay que mencionar que Julia, Emilio y Ezequiel emigraron a Cuba, siguiendo la trayectoria que había iniciado el propio Pedro y que continuó su hermano menor Ventura.

En efecto, Ventura Jado llegó a la isla de Cuba en 1846. El objetivo que perseguía era mejorar la situación económica, hacer fortuna, dedicándose al comercio. El viaje previo de su hermano Pedro y otras informaciones a las que accedió por relaciones de amistad le permitieron introducirse en una red en la que encontró protección y ayuda. Se instaló en La Habana y logró entrar como socio en un comercio llamado La Estrella, sito en la calle de la Muralla de la capital de Cuba.

La estrecha relación personal que vinculaba a los dos hermanos, lejos de atenuarse con la marcha de Ventura, se fortaleció gracias a la correspondencia cruzada entre ellos. Durante los primeros años de su estancia en la Isla la experiencia previa de Pedro sería un referente para su hermano, y hay que suponer que las cartas de uno a otro atravesarían el Atlántico con cierta frecuencia; sin embargo no se conserva ninguna anterior al año 1855.

En 1867, Emilio, hijo de Pedro, partió también hacia Cuba. Con su marcha el grupo familiar de los Jado Agüero en La Habana se potenció. A partir de este momento, Pedro Jado, consciente de que pertenecía a un grupo familiar que por las circunstancias se encontraba desgajado en dos lugares distantes, por su valor para mantener la cohesión del grupo, tomó la decisión de conservar la correspondencia. Las cartas unen a la familia y Pedro procuró preservarlas en la vivienda familiar estable. Es la razón que explica que, desde esta fecha, se incremente el número de cartas conservadas.

Cuando, pocos años más tarde, sus hijos Ezequiel y Julia se trasladaron también a Cuba, la decisión de escribir y conservar las cartas se mantuvo. Ezequiel Jado partió en tren hacia Cádiz en octubre de 1870; hay constancia de que en octubre de 1872 se encontraba en Cuba; trabajó primero en la tienda de ropas La Democracia, y en noviembre de 1873 entró a servir como Voluntario en la Compañía de Cazadores de La Habana, 5o batallón, de la que formó parte hasta junio de 1886.

Julia contrajo matrimonio con su tío Ventura en Escalante, y en diciembre de 1872 viajaron ambos a Cuba. Su adaptación a la isla antillana debió resultar difícil y falleció en el verano de 1873.

A la vista de la documentación que ha llegado hasta la actualidad podemos afirmar que Pedro Jado era un grafómano. Dedicaba a la escritura una cantidad considerable de su tiempo, a diario y con regularidad, en un lugar recogido de la casa al final de la jornada; en parte para registrar los datos relacionados con la administración de sus bienes, en parte para llevar la correspondencia que mantiene tanto en el círculo familiar como en el de los negocios. 
El conjunto epistolar más importante lo constituyen las cartas intercambiadas entre Pedro Jado y su hermano, Ventura, y los hijos del primero, Emilio y Ezequiel, que se hallaban emigrados en Cuba. La distancia, por una parte, y la importancia de los ingresos procedentes de la emigración, por otra, constituyen la base de esta relación epistolar tan intensa. El hermano de Pedro, Ventura, es el destinatario principal de sus cartas, no sólo por el gran número que le dirige, sino también por la frecuencia con que le escribe y el cuidado que pone en su redacción. A continuación se situan los dos hijos del matrimonio Jado-Ocejo que se encuentran con él en Cuba: Emilio y Ezequiel. Se han recopilado más de doscientos borradores y cartas intercambiadas entre estos cuatro corresponsales entre los años 1855 y 1883.

El intercambio entre otros miembros del núcleo familiar parece que fue mucho más reducido. No se puede, por el momento, saber si escribían también menos o si únicamente el número de cartas conservadas responde a azares del destino. En las cartas a Pedro Jado hay referencias a otros remitentes, a cartas escritas por otros miembros de la familia.

Pedro Jado era una persona concienzuda y metódica cuya puntualidad y cuidado al escribir y mantener su correspondencia es reconocida por sus amigos y familiares.

Querido tío: recibí su grata, fecha 3 del que rige, y he visto que tiene usted en su poder las tres cartas que yo creía perdidas, porque, como usted es tan puntual para todas las cosas, y no contestaba a las ya referidas, lo achacaba a su extravío o a falta de salud de esa familia (Carta de Valentín Lastra a Pedro Jado Agüero, Santander, 1869, Junio, 17).

Parece que era él el que escribía en mayor grado de la familia, pero puede ser también que fuera quien de todo el núcleo familiar tuviese mayor interés en conservar su correspondencia así como otros papeles. Una muestra de ese interés es el hecho de que mantuviese guardada su correspondencia en un envoltorio específicamente dedicado a este fin - "el envoltorio de la correspondencia" - y que él mismo menciona en una de sus cartas.

Mi estimado Juanito: tu padre me ha recordado, sin duda por tu indicación, creído que acaso no me habrían dado la tuya que me dirigiste con fecha 22 de abril último, que me entregaron oportunamente y que metí entonces en el envoltorio de la correspondencia sin contestarla (Carta de Pedro Jado a Juan Jorganes Lázaro, Escalante, 1884, junio, 18).

Asimilados a los miembros de la familia se pueden considerar criados y empleados de los Jado que se hallaban lejos de su lugar de origen, debido a distintas circunstancias, normalmente, el cumplimiento del servicio militar. Además de con diversos miembros de la familia, los Jado mantenían 
correspondencia de manera habitual con amigos y también con otras personas con las que estaban unidas por relaciones profesionales, comerciales y económicas.

La obligatoriedad de la correspondencia familiar se encuentra confirmada por las justificaciones ante la falta de correspondencia que se exhiben en las cartas. Continuamente se hace referencia a la falta de tiempo que ha impedido contestar o escribir algún mensaje y con frecuencia se explicita el motivo de la falta de puntualidad en el mantenimiento del correo.

No he sido más oportuno en contestar su carta, porque con la llegada de mi hermano Ventura, que tuvo lugar el domingo a la noche, no he podido ocuparme de ello hasta ahora (Carta de Pedro Jado a Florentino del Rivero, Escalante, 1862, Junio, 18).

Las muchas ocupaciones del corresponsal suelen ser aducidas como causa del retraso en la contestación de las cartas recibidas.

Mi apreciado amigo: recibí, por los mozos que fueron los dadores, su apreciada de usted fecha 30 del próximo pasado marzo y que no contesté por ser hora de la noche bastante avanzada. Y ahora que me encuentro algo desocupado, más que lo que he estado hasta aquí, dedico este momento a dar contestación a la suya citada (Carta de Pedro Jado a Juan J. Carasa, Escalante, 1871, Abril, sin día).

Muy señor mío y amigo: aunque el día tuviese 48 horas me faltaría tiempo para cumplir con todas mis obligaciones. Por eso no podré ni extenderme mucho ni escribir tantas veces como yo quisiera (Carta de José Rivero a Pedro Jado, Valladolid, 1874, Octubre, 20).

Las cartas cruzadas entre diferentes miembros de la familia Jado a ambos lados del Atlántico y las entradas personales en sus libros de cuentas se constituyen en una fuente documental excepcional donde se muestra la pulsión de la vida, los pensamientos, sentimientos y valores de sus autores $^{7}$. En ellas se pueden leer reflexiones sobre la emigración a América, la situación política, económica y militar en España, las guerras carlistas, la situación en Cuba y los movimientos insurgentes, pero también en relación con los aspectos centrales de este trabajo: la vida privada, los hábitos de alimentación, la higiene y la salud.

${ }^{7}$ Esta correspondencia se encuentra editada por Rosa M $M^{\underline{a}}$ Blasco Martínez y Carmen Rubalcaba Pérez (2003). En el texto se menciona el lugar y la fecha de cada misiva. 


\section{PREOCUPACIÓN POR EL ESTADO DE SALUD EN LA CORRESPONDENCIA}

La preocupación en torno al estado de salud de los diferentes miembros de la familia Jado aparece de manera repetida y en algunas ocasiones como único tema en las misivas ${ }^{8}$.

Lo que deseamos tu Mamá y yo es el que conserves la salud inalterable para ver si Dios te deja volver al país nativo para ver si podemos tener la dicha de volvernos a ver que son todos nuestros principales anhelos (Santoña, 1877 junio, 17).

Sólo cuando hay algún problema que momentáneamente capta la atención familiar, la salud pasa a un segundo plano, pero nunca desaparece. La inquietud originada por cualquier dolencia o malestar era natural en una época en el que la mortalidad provocada por enfermedades como la tuberculosis o el cólera era elevada. El clima tropical y las enfermedades endémicas de la isla de Cuba tenían un efecto muy negativo sobre la salud de los europeos que a ella llegaban causando la muerte de muchos de ellos. En la correspondencia se menciona en numerosas ocasiones el fallecimiento de jóvenes de la zona oriental de La Montaña, conocidos de la familia Jado, emigrados en la isla.

Si el interés por la salud de los familiares en Cuba era grande en periodos normales, cuando la situación en la isla se complica con una epidemia de cólera la angustia se apodera del resto de la familia en Escalante, que ruega noticias en cada correo y muestra con la indicación de sus rezos a San Roque el papel clave de la religión "a la hora de confrontar la muerte" (Zarzoso 2001: 430-431):

en esta seguimos bien, aunque con una pesadilla inexplicable por la mala noticia que acerca del estado sanitario de esa ha llegado por la vía de los Estados Unidos, de suerte que nos tiene esto disgustados, y por otro nos acompaña la confianza de que rogando á Dios por vosotros en nuestras cortas oraciones y a San Roque abogado contra la peste libraréis bien de tan fatal epidemia; nunca querido hermano he sido exigente en que escribáis con frecuencia pero ahora te suplico lo hagas todos los correos sin otra cosa más que el estado de salud en solo un renglón (Escalante, 1867 noviembre, 23).

De nuevo, en la despedida de la misma carta, Pedro Jado repite su súplica: "no te encargo otra cosa con mayor empeño que nos saquéis de penas todos los correos".

${ }^{8}$ Este aspecto aparece también en otras correspondencias familiares: "En la lectura de las cartas sorprende de inmediato confirmar la preocupación natural y cotidiana mostrada alrededor de la salud", Zarzoso (2001: 430). 
Uno de los hijos, Ezequiel, se ajusta en sus cartas a la petición expresada por su padre de escribir aunque fuese brevemente informando del estado de salud de los miembros de la familia en La Habana y envía misivas de muy corta extensión en los que se limita a mencionar que todos se encuentran bien. El joven se circunscribe a este argumento y lo resuelve en un par de líneas. En todas sus cartas, parece seguir una plantilla: se refiere a la salud de los que se hallan en Cuba y desea buena salud a sus padres y hermanos en Escalante.

Mi querido papá: sin ninguna de usted a que dirigirme; tomo la pluma para hacerle a usted sabedor de nuestra buena salud, y al ser ésta en sus manos se hallen ustedes gozando del mismo beneficio. De noticias del país nada de particular que le pueda mandar decir. Sin mas de usted muchas expresiones a todos los de casa de tío, Julia, Emilio y primos y usted reciba lo que guste de este querido hijo que verlos desea. Ezequiel Jado (Habana, 1873 febrero, 15).

Durante el siglo XIX y hasta principios del siglo Xx, persiste la influencia de un neohipocratismo vulgarizado que subraya los efectos del aire, el agua y la temperatura sobre la salud y que hace que las personas achaquen a la influencia del tiempo y de la estación los cambios en los diferentes ritmos fisiológicos (Corbin y Perrot 1989: 445). La teoría miasmática predominante en el panorama científico europeo del siglo XIX postulaba que las epidemias eran causadas por el estado de la atmósfera que deteriorada por la falta de condiciones higiénicas y sanitarias causaba la enfermedad, por ello la medicina del momento defendía la necesidad de las corrientes para limpiar el aire. La teoría contagionista que sostenía que las enfermedades infecciones se producían por gérmenes microscópicos fue, inicialmente, menos influyente en el mundo académico que la miasmática aunque finalmente logró imponerse (Martínez Hernáez 2008: 54).

Esta preocupación con respecto al agua, al sol y a las corrientes de aire queda reflejada en las cartas, donde se manifiesta el interés por el tiempo atmosférico y se recomiendan diferentes remedios para defenderse o paliar sus efectos sobre la salud?.

Pedro Jado menciona el beneficio que experimenta su salud con el calor, frente al empeoramiento que suponen los fríos invernales: "nosotros con

\footnotetext{
9 "La ignorancia de las causas de la fiebre amarilla y del paludismo, así como de otras "calenturas" en el siglo XIX, tenía su origen en la creencia arraigada en un determinismo climático cuya influencia en la salud de los habitantes era decisiva, argumentada en una teoría miasmática que concibe que las enfermedades son producto de emanaciones pútridas de las aguas, del aire y de sustancias orgánicas condicionadas por los cambios del tiempo atmosférico, especialmente por las lluvias y las altas temperaturas; es por ello que se habla de "calenturas estacionales"” (Figuera von Wichmann 2009: 153).
} 
los calores lo pasamos ahora menos mal que en el invierno porque el frío para los viejos es lo que más sienten y nosotros participando ya de esta enfermedad y no puedes suponer cual puede ser nuestro estado; y aún así quisiera uno vivir hasta ser sacado con carpancho al sol, no sé qué tiene esto de la muerte que tanto la siente todo ser viviente" (Santoña, 1877 junio, 17). El calor es media vida, en palabras del mismo Pedro Jado, y "con el alimento vida entera como suele decirse” (Santoña, 1879 agosto, 17).

Mi querido hijo Emilio: con el placer que puedes figurarte, recibí tu última de 25 de julio que nos entera de tu buena salud; en ésta por hoy sin novedad todos, aunque tu mamá y yo sentimos los fríos que se advierten por las aguas tan abundantes que hace días tenemos y las noches que han alargado y que impresionan demasiado a los de avanzada edad como nosotros; pero siempre así y nunca peor como suele decirse, a Dios gracias (Carta de Pedro Jado a su hijo Emilio. Santoña, 1880 setiembre, 17).

\section{TRATAMIENTOS MÉDiCOS ESENCIALES}

Durante el periodo histórico en el que se desarrolla la vida de Pedro Jado, los tratamientos médicos esenciales giraban en torno a prácticas como las purgas, regímenes dietéticos, reposo, baños, sangrías, aplicación de sanguijuelas, ventosas, purgantes o enemas que se inscribían en la visión médica del momento centrada en la distribución de los distintos humores. Las sangrías, herencia también de la tradición galénico-hipocrática se constituyen a lo largo del siglo XIX, como en periodos anteriores, en un recurso terapéutico habitual. En la correspondencia y en diferentes entradas de los libros de cuentas de la familia Jado se hace mención en numerosas ocasiones a su utilización.

El día 24 de Enero de 1856 vino después de la hora de las doce del día Don Ramón Cobo y sangró a Joaquina según queda referido, comió y entre tres y cuatro de la tarde marchó, la sangría solo fue a prevención mediante estar embarazada (Libro de cuentas, 24 de enero de 1856).

En la tarde del día 10 de Abril de 1856 a buscar a Don José de la Lastra cirujano sangrador sin título y vino con el criado y se quedó por la noche en casa con motivo de unos retientos de dolores de parto que le dieron a Joaquina al día siguiente después de tomar chocolate se fue como que no fue necesario para nada y dijele al marchar correspondería con él cuando volviera que dentro de pocos días sería necesario a la hora menos pensada (Libro de cuentas, 10 de abril de 1856).

En Julio 1 por la tarde sangró el cirujano Lavín a Bernabé; y visto se seguía el dolor del costado al día siguiente se mandó a buscar a Revuelta á Beranga, vino y le sangró por la tarde (Libro de cuentas, 1 de julio de 1856). 
Otro remedio médico habitual procedente de la tradición hipocrática era la aplicación de extracto de cantárida. En el Formulario enciclopédico de medicina, farmacia y veterinaria (1889-1893) de Mariano Pérez M. Minguez se describe el uso de este insecto en medicina: "De todos los epispáticos, este insecto es el más usado porque su acción principal se ejerce sobre la piel, por más que los principios activos pueden ser absorbidos y causar graves accidentes".

Don Casimiro Menezo me hizo varias visitas, entre ellas una que creo fue la víspera de San Emeterio a las doce o una de la madrugada que fue el que recetó las cantáridas, por lo que débole doble atención (Libro de cuentas, agosto, 1856).

El sábado 23 de agosto de 1856 fue el primer día que hice cama de la fiebre que he tenido, el domingo 24 a la mañanita vino Don Lázaro Valle y me sangró, volvió por la noche y me sangró a las 8 , volvió el lunes a la mañanita y me volvió a sangrar, todas tres sangrías me las dio del brazo izquierdo, a los pocos días después me pusieron 24 sanguijuelas en la boca del estómago. El día de San Emeterio por la noche o la noche antes dos sinapismos en las pantorrillas y después que levantaron estos, dos cantáridas una en cada pantorrilla; no recuerdo a los cuantos días del mal se me presentó un sudor fuerte que principió a las once del día y seguí copiosamente sudando el resto del día toda la noche hasta el amanecer pues aunque todo el día siguiente estuve también fue más suavemente; me levanté el primer día 9 de septiembre a las once de la mañana a los 18 días de cama (Libro de cuentas, septiembre, 1856).

\section{LA HIDROTERAPIA COMO RECURSO}

De manera paralela, y frente al azote de la tuberculosis, se despliegan curas basadas en remedios naturales como eran los cambios de aires, los baños termales o marinos y el reposo (Martin-Fugier 1989: 238).

El recurso a las aguas tanto minerales, termales como marinas es continua entre los diferentes miembros de la familia Jado. Respecto a los baños en el mar, que empezaron a conocer gran éxito en el norte de España a partir de la presencia de la familia real en Santander en la segunda mitad del siglo XIX, numerosos familiares y amigos de los Jado se trasladan a esta región para tomarlos ${ }^{10}$. Así escribe Pedro Jado a su hermano Ventura:

10 "[...] la reputación que alcanzaban los "baños de ola" como eficacísimo remedio terapéutico y la indudable belleza del paraje atraían ya, en 1840, a numerosos santanderinos, temprana y justamente enamorados de su paisaje, cuyo número se acrecentó con quienes llegaban de otras provincias impulsados por los mismos atractivos [...] Ya en 1852 había visitado nuestra provincia el Rey don Francisco de Asís para inaugurar las obras del ferrocarril de Alar, y en 1861 fue la propia Reina Isabel quien decidió sumergirse en las olas del Sardinero para aliviar el herpes que padecía" (Rodríguez Alcalde 1991: 7-8). 
"Gerardo y Petra que han estado en la montaña, bañándose ambos de agua salada, cerca de dos meses, marchan mañana para Zamora" (Santoña, 1876 setiembre, 4).

El uso de aguas minerales con fines curativos se había difundido desde finales del siglo XVIII alcanzando un gran desarrollo en el siglo XIX, viéndose apoyada la generalización de la práctica balnearia por la propagación del higienismo que considera decisiva la influencia del entorno ambiental y social para la formación de procesos patológicos y en consecuencia, también para su curación (San Pedro Martínez 1993: 31). En la antigua provincia de Santander existían numerosos balnearios y diversos miembros de la familia Jado hacen uso de ellos. Acuden a Liérganes, Solares, Alceda, Caldas de Besaya y mencionan en sus cartas las estancias en estos lugares:

En ésta todos bien, pues tu sobrina se ha puesto muy mejorada en su salud, hace pocos días vino con su madre que la fue acompañar de las aguas de los baños de Liérganes que las recomendaron los facultativos y la han probado bien hasta ahora (Escalante, 1883 setiembre, 17).

De maneras diversas se llevaba a cabo el uso y aplicación de estas aguas minerales: baños, ingestión o inhalación de vahos. Así, a uno de los hijos de Pedro Jado, Mariano, un médico de Santander le recomienda beber agua de Solares y según Pedro Jado, "éstas le han limpiado el estómago por ser purgantes y ha recobrado el apetito y come bastante bien" (Escalante, 1879 julio, 18). Pedro Jado escribe a su hijo Ezequiel y le dice: "te dirijo la presente para decirte que Mamá, Venturín y Atanasia han estado a los baños de Alceda de donde han regresado el ocho del corriente y continúan regular menos Venturín que éste no ha tomado baños generales, sino vahos y las aguas le ha probado bastante bien. Mamá no se si tendrá que volver en septiembre, bien mal tendrá que verse, porque cualquier salida origina muchos gastos" (Santoña, 1877 julio, 17).

La visita a los centros termales se constituye en una tradición y año tras año se acude a ellos en busca de alivio: "Estamos en esta desde primeros del corriente que vinimos a recoger la hierba de unos praditos que no pude arrendar y continuaré aquí hasta que Joaquina y Venturín vuelvan de los baños de Alceda á donde fueron el año pasado y les probaron bastante bien" (Escalante, 1878 julio, 17). También Pedro Jado prueba los baños: "Yo he estado en los baños de las Caldas de Besaya, y por los cuales voy consiguiendo algún alivio aunque muy lento” (Escalante, 1868 julio, 8).

A finales del siglo XIX, el cambio de aires, especialmente hacia regiones de montaña o más templadas, se prescribía con frecuencia para intentar frenar los estragos de la tuberculosis que, aunque no se menciona por su nombre en ninguna ocasión, clavó sus garras en varios de los miembros más jóve- 
nes de la familia. Refiriéndose a uno de sus hijos, Mariano, afectado también por una tos muy fuerte y dolores en el costado, escribe Pedro Jado a su hermano Ventura: "había concebido hace como un mes y medio a dos que mi hijo Mariano aunque con convalecencia larga se restablecería completamente en su salud, pero hoy un nudo oprime mi corazón al verle padecer de nuevo y aunque se levanta todos los días la tos se le ha vuelto a recrudecer con algunos dolorcillos que siente de medio cuerpo arriba" (Santoña, 1879 abril, 17). Como a pesar de todas las medidas adoptadas, Mariano no mejoraba en su enfermedad se decidió que se trasladara fuera de la región y, enfermo gravemente y sin apenas esperanzas de curación, se desplaza hasta Panticosa en Huesca.

Esta sirve para saludarte y poner en tu conocimiento desgraciadamente que Mariano sigue muy mal, pues el 6 y 13 del corriente ha tenido dos vómitos de sangre tan fuertes que nos ha llenado de consternación y susto por haber echado muchísima sangre en ambas veces, y le ha dejado sumamente extenuado y delicado, a lo que se le agrega la ninguna gana de comer que tiene; así es que estamos sumamente disgustados y sin humor para nada. Él está bastante animado para ver si se puede poner en disposición de salir a las aguas de Panticosa que tan bien le probaron el año pasado, pero dudo que pueda ponerse en disposición de salir de casa; él todos los días se levanta pero no puede salir del cuarto (Carta de Pedro Jado a Emilio, Santoña, 1880, junio, 17).

\section{MEDIDAS HIGIÉNICAS Y DIETÉTICAS ADOPTADAS}

De manera paulatina, se desarrolla en toda Europa un proyecto higiéni$\mathrm{CO}^{11}$, que abraza no sólo la higiene corporal y todo un conjunto de prescripciones que intentan instaurar un orden preciso en la forma de vida (diversiones, ejercicios, emociones...), sino también una higiene alimenticia y una completa dietética.

Distintos remedios caseros son intercambiados a través de las cartas. Lo que indica que "el primer nivel de la cultura médica estaba constituido por los consejos de la familia y las amistades" (Zarzoso 2001: 430). Ventura Jado, por ejemplo, escribe a su hermano y le recomienda una cura para su sobrino Alberto cuya tos no cesaba de empeorar. En la misiva destaca que él mismo ha probado el remedio aconsejado ${ }^{12}$.

11 "Uno de los rasgos más sobresalientes de la literatura médica de divulgación en el siglo XIX es la preocupación que manifiesta por los temas de higiene y las condiciones de vida de las clases populares" (Fuentes Caballero 1997: 239. Véase también González Castillejo 1997: 251-259).

12 "Hay que subrayar la importancia de la experiencia personal cuando se comparten remedios o se afirma la utilidad de un tratamiento determinado" (Zarzoso 2001: 432). 
Como pudiese suceder que fuese algún catarro pasmado, de haberlo cogido y abandonado sin sudarlo y echarlo, os voy a dar un remedio que no es nocivo y está probado hace mucho bien para los catarros aunque sean viejos, es el siguiente: en una chocolatera se pone una yema de huevo, unas tres cucharadas sobre grande de azúcar quebrada; esta yema y el azúcar ya citado se deslíe perfectamente con un morenillo, y cuando esté bien desleído el azúcar y la yema de huevo se va echando agua muy caliente en la chocolatera, y cuando esté cayendo el agua en la chocolatera — que se echará poco a poco— el morenillo que esté batiendo como si estuviesen haciendo chocolate. Se echará una cantidad de agua como para un vaso grande que tomará al acostarse todo lo caliente que pueda, y enseguida abrigarlo bien cosa de que sude por cada pelo mil gotas; este remedio sí ha dado muy buenos resultados, incluso en mi persona; debe tomarse ocho noches seguidas, al acostarse, y tomarlo todo lo más caliente que se pueda, sentado ya en la cama, y enseguida abrigarlo bien y a sudar (Habana, 1876 noviembre, 15).

La dietética, junto con la cirugía y la farmacoterapia, componía la "trina ordenación de Celso" en la que se basaban prácticamente todos los tratamientos médicos para los integrantes de las clases sociales que podían permitírselos, a lo largo del siglo XIX (Figuera von Wichmann 2009: 153). Siguiendo la máxima hipocrática, coincidente a su vez con el sentir popular, que afirmaba que el alimento era la mejor medicina, la familia Jado proporciona a los enfermos de la casa aquellos platos considerados beneficiosos, como caldos, leche y chocolate ${ }^{13}$.

El día 11 de Julio de 1856 se mató por la tarde un pollo para poner puchero para darle el primer caldo a Bernabé, mi criado, del mal que tiene y le dio el 30 de junio al anochecer (Libro de cuentas, 11 de Julio de 1856).

El consumo de leche fresca estaba recomendado en situaciones de enfermedad o debilidad (Pujol, Nicolau y Hernández 2007: 310).

[...] nos encontramos divididos Joaquina con Mariano está en Escalante va hacer como un mes aproximadamente para tomar éste leche de cabra alternada con la de burra (De Pedro Jado a su hermano Ventura, Santoña, 1880 abril) ${ }^{14}$.

De igual manera, desde su llegada a Europa desde América hasta el siglo XIX, el chocolate está considerado un medicamento y por ello se intenta disponer de él en el hogar (Huetz de Lemps 2004: 784-785). Para ello, Pedro Jado paga a un chocolatero de una localidad vecina para que lleve a cabo la molienda de chocolate: "El chocolatero de Meruelo vino hoy por la

13 "El padre de la enfermedad pudo haber sido cualquiera, pero sin duda la madre es la mala dieta. Que el alimento sea tu mejor medicina y tu mejor medicina sea tu alimento" (citado por Torres Aured 2009: 271).

${ }_{14}$ Cuartilla en muy mal estado de conservación, no se puede apreciar la fecha completa. 
mañana a hacerme la molienda de chocolate" (Libro de cuentas, 20 de Junio de 1855).

Los diferentes estudios, históricos y etnográficos, sobre la salud en el siglo XIX muestran el predominio de la idea de la moderación y el rechazo de los excesos como condiciones para el mantenimiento del bienestar y el logro de una vida saludable. Las emociones fuertes se consideran causa de enfermedades varias y se recomienda contra ellas remedios como las sangrías. En noviembre de 1878, la casa donde vivían los Jado en Santoña sufrió un incendio, a consecuencia del cual escribe Pedro Jado: "la criada también ha tenido que marcharse, pues se debió sangrar al día siguiente, no lo hizo y se halla enferma en su casa por consecuencia del susto" (Santoña, 1878 noviembre, 18).

FE Y RESIGNACIÓN: VALORES FAMILIARES DE LOS JADO

Muy unida a la idea de la moderación como objetivo vital se encuentra el mantenimiento de una conducta moral intachable. La familia no es únicamente un conjunto de personas unidas por lazos de parentesco y ligados - en el caso de familias burguesas o acomodadas- a un patrimonio o solar, sino que es también un "capital simbólico de honor" (Perrot 1989: 272). Todo aquello que manche la reputación de uno de sus miembros, empaña la honra de la familia. Por este motivo son continuas las alusiones y las recomendaciones de Pedro Jado en torno a la necesidad de que sus hijos observen una conducta ejemplar, puesto que "el hombre debe sujetar las pasiones que son contrarias al bien obrar, y recapacitar mucho sobre su buen modo de proceder en todo para ser estimado y querido de cuantos le traten" (Santoña, 1874 junio 11). Para lograrlo, Pedro Jado, recomienda a sus hijos acudir a la fe: Te aconsejo no olvides á Dios y la Virgen por la mañana y noche, dirigiéndoles la oración siguiente: Alabada sea la Santísima trinidad Padre, Hijo y Espíritu Santo, tres personas distintas y un solo Dios verdadero, el cual me alumbre a mí y eche su bendición en este día, librándome de todo mal, y por intercesión de su Madre Santísima me lleve a su santa gloria. Amen. Ave María (Santoña, 1874 junio, 12).

La enfermedad tiene un significado colectivo y en este momento, siglo XIX, se engrana dentro de los valores católicos de resignación ante los padecimientos como muestra de la voluntad divina y súplica a la misma para que, si lo desea, cese en ellos. La idea de supeditación de la vida del individuo a la voluntad divina se encuentra difusa en la sociedad decimonónica y se reconoce en las manifestaciones populares de religiosidad como rogativas, exvotos y ofrendas. Durante la epidemia de cólera del año 1834 en la ciudad de Santander, es el propio cabildo del ayuntamiento el que ordena celebrar rogativas durante tres días y una procesión claustral para implorar 
de la misericordia divina el fin de la plaga (Maestre Sánchez 1985: 149). En este periodo se desarrolla un fuerte movimiento de devoción a la Virgen, a cuya protección, como se ha visto, se encomienda y encomienda a sus hijos con frecuencia Pedro Jado ${ }^{15}$.

Con ocasión de una epidemia de cólera en el año 1855, Pedro Jado describe en su libro de cuentas las distintas iniciativas adoptadas en su población para rogar por el fin de la misma.

Hubo rogativas a San Roque con misa y rosario y se seguirá un novenario cantado desde hoy [...]. Esta rogativa y novenario fue acordado se hiciera para alcanzar del glorioso San Roque que interponga su valimiento con el Señor para que nos libre del azote del cólera que se dice se ha desarrollado en la ciudad de Santander (Libro de cuentas, 17 octubre 1854).

El día 11 de Septiembre de 1855 se celebró una misa [...] con intención y ofrecida para que interponga San Roque su influjo con Dios para que nos dé salud espiritual y temporal si nos conviene y nos libre del azote de la peste que reina; otra misa tan solemne se celebrará tan pronto calme esta peste en acción de gracias (Libro de cuentas, septiembre 1855).

El día 19 de septiembre de 1855 se canto el Te Deum por haber cesado la epidemia del cólera en Santa Clara, por la tarde se rezó el rosario en las calles y sacaron á Nuestra Señora de la Cama [en Escalante] (Libro de cuentas, Septiembre 1855).

Junto a la fe, la resignación - ligada estrechamente a la primera, en la convicción de que lo que sucede es voluntad de Dios- es una constante en la moral de la familia Jado, propia por otra parte, de las familias católicas del siglo $\mathrm{XIX}^{16}$.

En una de sus cartas Ventura le dice a su hermano Pedro: "La lotería no nos quiere favorecer, prueba de que no nos conviene" (Habana, 1878 enero, 15). Y sobre el mismo tema de la lotería vuelve a escribirle en fechas posteriores: "En el billete número 8.360 de la lotería extraordinaria que

15 "La cronología de la edad mariana de la Iglesia católica queda aproximada y simbólicamente acotada por la definición de dos controvertidos dogmas relativos a la Virgen. El primero, proclamado en 1854 por el papa Pío IX, afirmaba la Inmaculada Concepción de María; el segundo, definido en 1950 por Pío XII, confirmaba la Asunción de María al Cielo. Entre ambas fechas, las apariciones marianas [...], las peregrinaciones marianas [...], las cofradías y congregaciones marianas, las oraciones marianas y muchas otras formas de devoción a María marcaron e impulsaron la renovada vitalidad del catolicismo durante los siglos XIX y XX" (Cueva Merino 1999: 964).

${ }^{16}$ En su trabajo sobre el pluralismo médico en la Cataluña del siglo XVIII, Zarzoso (2001: 432) afirma: "Los autores de las cartas aceptan con resignación cristiana la enfermedad y la muerte, como el producto de la divina voluntad, lo que les lleva a identificar abiertamente la enfermedad con su condición de pecadores. Sin embargo, este hecho no les impide luchar contra la enfermedad y recurrir a todos los resortes disponibles para intentar restablecer su salud". 
se ha de celebrar el día 13 del corriente jugáis tú y Joaquina un vigésimo, y Joaquinita, Darío y Venturín otro vigésimo, a ver si Dios quiere que nos saquemos el millón si es que nos conviene" (Habana, 1878 abril, 5). De nuevo, ante otra desgracia familiar, la muerte de Mariano, Pedro Jado en carta a su hijo Emilio escribe: "Dios quiera mejorar las horas si nos conviene” (Santoña, 1880 setiembre, 17).

Si a la enfermedad se hace frecuente referencia en la correspondencia de la familia Jado, cuyos miembros se extienden en la descripción de trastornos, cólicos, enfermedades y sus subsiguientes remedios, caseros o médicos, hasta extremos que hoy pueden resultar sorprendentes, la muerte resulta menos citada. Se menciona el fallecimiento de conocidos y familiares lejanos, pero en el momento de hablar de los familiares más cercanos, la pluma parece detenerse y se pasa sobre ello con rapidez. Se escribe con profusión sobre la enfermedad y sus tratamientos, pero hay un gran pudor a la hora de tratar el dolor de la muerte.

[hemos] padecido muchísimo con el pobre tu hermano en los tres últimos meses de su vida que tanto que padeció y padecimos todos al verle sufrir especialmente un mes antes de su muerte cuanto más malo se sentía más anhelos demostraba por vivir y esto aumentaba la pena de todos y al tener el segundo vómito de sangre ya él mismo nos dijo conformidad que él se marchaba; cabiéndonos la grata satisfacción de haberse dispuesto perfectamente confesándose y recibiendo a Dios con tan buena fe (Santoña, 1880 setiembre, 17).

\section{LAS ESCRITURAS DEL YO COMO ESPACIO PARA CONOCER LA INTIMIDAD DEL PASADO}

Los significados de la salud y de la enfermedad están social y culturalmente construidos. Son formas de la realidad social, constituida por y creadora a su vez de significados, instituciones y relaciones, sancionados o legitimados por la sociedad (Kleinman 1980: 35-36).

La enfermedad no consiste sólo en una disfunción biológica, sino también en una matriz de significados culturalmente establecidos que hay que intentar desentrañar. Descubrir el sentido simbólico de la enfermedad - a través del análisis del conjunto de experiencias, de palabras y de sentimientos utilizados o descritos por los integrantes de un grupo cultural- significa acercarse en mayor medida al conocimiento de la estructura de significados de esa sociedad.

Revelar los sentidos escondidos en los aparentes es una tarea intelectual que debe permitir esclarecer los vínculos entre las representaciones de la enfermedad y sus significados culturales. Por ello, el estudio de la visión personal y social de la salud y la enfermedad, así como de los tratamientos terapéuticos o los remedios populares, presenta tan gran interés para poder 
profundizar en el conocimiento del imaginario social del momento. En palabras de Ángel Martínez Hernáez (2008: 8), "los padecimientos humanos constituyen universos narrativos y simbólicos que condensan formas de experiencia propias de un mundo local".

En el caso de la familia Jado dos variables - la corriente neohipocrática predominante en la medicina de la época y su fe cristiana- influirán en su concepción de la salud y la enfermedad y sobre todo en su actitud ante ellas y ante la muerte. La continua sumisión a la voluntad de la divinidad refleja la profunda religiosidad de la familia que se exterioriza a través de rituales, oraciones y actitudes.

Pedro Jado y sus familiares atribuyen sentido a la enfermedad y a la muerte, intentan hacerla inteligible por medio de la narrativa en el discurso autobiográfico y epistolar. A través de un acercamiento antropológico a sus testimonios de cultura escrita es posible acercarse a las concepciones y visiones socioculturales del momento, puesto que "la perspectiva personal es sólo una lente a través de la cual se percibe lo universal" (Durán Giménez Rico 1999: 106).

Las escrituras del yo se constituyen como un espacio privilegiado para una búsqueda de la identidad del individuo ${ }^{17} \mathrm{y}$, por ende, de su grupo humano de pertenencia — no en vano Montaigne afirma que cada hombre lleva en sí la forma entera de la humana condición (Gusdorf 1975: 978) — y a través de ellas, se alcanza en ocasiones el mejor conocimiento de la intimidad en el pasado, uno de los objetivos más difíciles de lograr en el estudio histórico, y de diversos aspectos de la vida humana como, en este caso, la visión de la enfermedad.

Cuando el ser, la propia existencia, está en juego el papel se convierte en soporte espiritual de la persona. De esta manera, redactar, ordenar un texto se convierte en una forma de organizar la propia experiencia vital y de transmitirla a otros, de interpretarla y extraer de ella conclusiones. A través de la narración del padecimiento, el enfermo hace posible el acceso a su vivencia íntima de la enfermedad en oposición a la enfermedad construida desde fuera, en especial por el sistema médico.

Los escritos personales, íntimos, nos permiten conocer la biografía de un ser humano que vive dentro de una sucesión histórica y, como escribió Ch. W. Mills (1964: 26), "ningún estudio social que no vuelva a los problemas de la biografía, de la historia y de sus intersecciones dentro de la sociedad, ha terminado su jornada intelectual".

17 "Livres du Moi, Selfsbooks, Selbstzeugnisse composent, au cœur de la littérature d'Occident, une région privilégiée, foyer d'animation et centre d'interprétation pour une recherche de l'identité à la faveur de laquelle l'être humain se propose de s'annoncer à lui-même, et d'annoncer aux autres, dans certains cas, la vérité intrinsèque de ce qu'il est" (Gusdorf 1991: 9). 


\section{BIBLIOGRAFÍA CITADA}

Ackerknecht, E. H. 1945. Malaria in the Upper Mississippi Valley, 1760-1900. Baltimore: Johns Hopkins University Press.

Ackerknecht, E. H. 1963. Geschichte und Geographie der wichtigsten Krakenheiten. Stuttgart: F. Enke.

Ackerknecht, E. H. 1971. Medicine and Ethnology. Bern: Huber.

Ackerknecht, E. H. 1982. A short history of medicine. Baltimore: Johns Hopkins University Press.

Anderson, M. 1988. Aproximación a la historia de la familia occidental (1500-1914). Madrid: Siglo XXI.

Arrizabalaga, J. 1992. "Nuevas tendencias en la historia de la enfermedad: a propósito del constructivismo social". Arbor, CXLII, 558-559-560: 147-165.

Blasco Martínez, R. M. y C. Rubalcaba Pérez. 2003. "Para hablarte a tan larga distancia...": correspondencia de una familia montañesa a ambos lados del Atlántico (18551883). Santander: Estudio.

Burguière, A. y otros (dirs.). 1988. Historia de la familia. Madrid: Alianza Editorial. 2 vols.

Chartier, R. 1991. "Avant-propos", en R. Chartier (dir.), La correspondance. Les usages de la lettre au XIX siècle. París: Fayard.

Comelles, J. M. 2002. "Luis García Ballester, antropólogo". Dynamis. Acta Hispanica ad Medicinae Scientiarumque Historiam Illustrandam 22: 487-492.

Corbin, A. y M. Perrot. 1989. "Entre bastidores", en P. Ariès y G. Duby (dirs.), Historia de la vida privada. T. 4, De la Revolución Francesa a la Primera Guerra Mundial: 419-617. Madrid: Taurus.

Cueva Merino, J. de la. 1999. "La construcción de una identidad católica regional: La Bien Aparecida patrona de La Montaña", en I Encuentro de Historia de Cantabria, Vol. 2: 963-981. Santander: Universidad de Cantabria.

Dauphin, C. ; P. Lebrun-Pézerat y D. Poublan. 1995. Ces bonnes lettres : une correspondance familiale au XIX siècle. París: Albin Michel.

Duden, B. 1991. The woman beneath the skin. A doctor's patients in eighteenth-century Germany. Cambridge-Mass.: Harvard University Press.

Durán Giménez-Rico, I. 1999. "El género autobiográfico en la literatura inglesa: Gran Bretaña y Estados Unidos", en M. Ledesma Pedraz (ed.), Seminario "Escritura Autobiográfica": II Seminario Escritura autobiográfica: 99-118. Jaén: Universidad de Jaén.

Figuera von Wichmann, E. de la. 2009. "Las enfermedades más frecuentes a principios del siglo XIX y sus tratamientos", en M. L. Torres Aured (coord.), Los sitios de Zaragoza: alimentación, enfermedad, salud y propaganda: 151-171. Zaragoza: Institución Fernando El Católico.

Fuentes Caballero, M. T. 1997. "El discurso sobre la lactancia en la literatura médica de final del siglo XIX. Barcelona 1880-1890", en R. Rowland e I. Moll Blanes (eds.), La demografía y la historia de la familia: 239-249. Murcia: Universidad de Murcia.

González Castillejo, M. J. 1997. "El discurso higienista como instrumento de control social y cohesión familiar en el primer tercio del siglo Xx", en R. Rowland e I. Moll Blanes (eds.), La demografía y la historia de la familia: 251-259. Murcia: Universidad de Murcia.

Gusdorf, G. 1975. "De l'autobiographie initiatique à l'autobiographie genre littéraire". Revue d'Histoire Littéraire de la France LXXV (6): 957-994. 
Gusdorf, G. 1991. Lignes de vie 2: auto-bio-graphie. París: Éditions Odile Jacob.

Huetz de Lemps, A. 2004. "Bebidas coloniales y auge del azúcar", en J-L. Flandrin y M. Montanari (dirs.), Historia de la alimentación: 781-795. Gijón: Trea.

Kleinman, A. 1980. Patients and healers in the context of culture: an exploration of the borderland between anthropology, medicine, and psychiatry. Berkeley: University of California Press.

López Piñero, J. M. y M. L. Terrada Ferrandis. 2000. Introducción a la medicina. Barcelona: Crítica.

Maestre Sánchez, A. 1985. El cólera en Santander: la epidemia del año 1834. Santander: Ayuntamiento de Santander.

Martínez Hernáez, Á. 2008. Antropología médica: teorías sobre la cultura, el poder y la enfermedad. Madrid: Anthropos.

Martin-Fugier, A. 1989. "Los ritos de la vida privada burguesa", en P. Ariès y G. Duby (dirs.), Historia de la vida privada, T. 4, De la Revolución Francesa a la Primera Guerra Mundial: 199-267. Madrid: Taurus.

Mills, C. W. 1964. La imaginación sociológica. México: Fondo de Cultura Económica.

Perrot, M. 1989. "Dramas y conflictos familiares", en P. Ariès y G. Duby (dirs.), Historia de la vida privada, T. 4, De la Revolución Francesa a la Primera Guerra Mundial: 269-291. Madrid: Taurus.

Porter, R. (ed.). 1983. Patients and practitioners. Lay perceptions of medicine in pre-industrial society. Cambridge: Cambridge U.P.

Porter, R. 1985. "Doing medical history from below". Theory and Society 14: 175-198.

Porter, R. y A. Wear (eds.). 1987. Problems and Methods in the History of Medicine. Londres: Croom Helm.

Pujol Andreu, J.; R. Nicolau Nos e I. Hernández Adell. 2007. "El consumo de leche fresca en Cataluña entre mediados del siglo XIX y 1935: la difusión de un nuevo alimento". Historia agraria: Revista de agricultura e historia rural 42: 303-325.

Rodríguez Alcalde, L. 1991. Crónica del veraneo regio. Santander: Ediciones de Librería Estudio.

Rosen, G. 1943. The History of Miners' Diseases, A Medical and Social Interpretation. Nueva York: Schuman.

San Pedro Martínez, M. A. 1993. El balneario de Puente Viesgo (1796-1936). El turismo balneario de interior en Cantabria. Génesis, esplendor y decadencia de un espacio de ocio. Santander: Fundación Marcelino Botín.

Sigerist, H. E. 1943. Civilisation and Disease. Ithaca: Comell University Press

Torres Aured, M. L. 2009. "Alimentación y cuidados desde San Juan de Dios a la Madre Rafols y un posterior tratado recopilatorio", en M. L. Torres Aured (coord.), Los sitios de Zaragoza: alimentación, enfermedad, salud y propaganda: 245-288. Zaragoza: Institución Fernando El Católico.

Zarzoso, A. 2001. "El pluralismo médico a través de la correspondencia privada en la Cataluña del siglo XvIII". Dynamis. Acta Hispanica ad Medicinae Scientiarumque Historiam Illustrandam 21: 409-433. 